\title{
BIM 技术在市政路桥设计施工方面的应用浅析
}

\author{
王 振 张保坤 \\ 中国市政工程华北设计研究总院有限公司，天津 300202
}

[摘要] 随着我国经济的不断发展, 市政道路桥梁建设规模及数量也逐年递增, 传统设计中的不足之处也逐渐显现出来; 文章 中对 BIM 技术进行了简要的介绍说明，通过论述其在设计中的优点，体现出了该技术在路桥设计行业中的优势与特点; 又对 现阶段 BIM 技术的发展进行了一定的介绍，在此基础上列举了 BIM 技术的具体应用; 最后对 BIM 技术的发展进行了展望。 [关键词]BIM 技术; 道路桥梁设计; 设计优化; 数字化; 三维设计

DOI: $10.33142 /$ ec.v3i3.1604

中图分类号: U442.5

文献标识码：A

\section{Application of BIM Technology in the Design and Construction of Municipal Roads and Bridges}

WANG Zhen, ZHANG Baokun

North China Municipal Engineering Design \& Research Institute Co., Ltd., Tianjin, 300202, China

\begin{abstract}
With the continuous development of China's economy, the construction scale and quantity of municipal roads and bridges are increasing year by year, and the shortcomings of traditional design are gradually emerging. In this paper, the BIM Technology is introduced briefly. By discussing its advantages in design, the advantages and characteristics of BIM Technology in the road and bridge design industry are reflected. At the same time, the development of BIM Technology at present is introduced. On this basis, the specific application of BIM Technology is listed. Finally, the development of BIM Technology is prospected.
\end{abstract}

Keywords: BIM Technology; road and bridge design; design optimization; digitalization; 3D design

引言

随着信息化及数字化的不断进步与发展, 各行各业逐渐开始实现数字化, BIM (Building Information Modeling) 的出现为路桥设计、施工的数字化提供了方向; 因此, BIM 技术成为了当前道路桥梁设计关注的重点, 该技术是将信息技 术与科学技术应用在道路设计中, 增加了道路设计的科学性与准确性; BIM 技术的应用应不仅可以提高路桥设计的效率与 质量, 还能提供更为直观的立体效果, 对于道路、桥梁后期施工及运营等阶段也有着重要意义。BIM 技术在建筑行业应用 较为广泛, 市政路桥行业也有着良好的发展前景, 随着技术的不断发展与完善, 将会促进整个行业的提升与进步。

\section{BIM 技术简介}

BIM (Building Information Modeling) 是指建筑信息模型, 其核心是通过建立虚拟的建筑工程三位模型, 利用 数字化技术，为这个模型提供完整的、与实际情况一致的建筑工程信息库。

早在 20 世纪 70 年代至 90 年代, BIM 的理念就已经提出, 美国 Chuck Eastman 教授提出了 “Building Description System” 的工作系统原型, 被称为 “BIM 之父”, 由于当时计算机技术发展较为缓慢, BIM 技术也仅仅停留在了理论研 究阶段, 并未进行深入。

BIM 技术是 Autodesk 公司在 2002 年率先提出, 现阶段在全球范围内得到了业内的广泛认可, 随着计算机技术的不 断革新, BIM 软件也逐渐增多, 较为常见的软件有 Autodesk、ArchiCAD、Bentley 等。

美国的 BIM 技术研究与使用开始较早, 目前, 美国大多数建筑已经开始应用 BIM 技术, 且 BIM 技术标准相对完善; 欧洲、日本、新加坡等国家的 BIM 发展和应用也达到了较高水准; 相比国外 BIM 技术的应用与发展较为落后, 仅在建 筑行业有所应用, 市政路桥等行业 BIM 技术的应用刚刚起步, 并未形成体系, 相关软件也还没有得到广泛推广与认同, BIM 技术尚处于探索阶段, 以后的发展还有很长的路要走 ${ }^{[1]}$ 。

\section{BIM 技术在道路桥梁设计中的优点}

2.1 现阶段道路设计中存在的问题

我国市政路桥设计的方法主要为计算机 (CAD) 辅助设计; 计算机 CAD 的出现使图纸设计由手绘阶段发展到了计算 机绘图阶段, 这次行业的变革大大加快了设计速度, 提高了设计的准确性; 但是计算机绘图依旧是二维绘图, 并没有 解决二维图纸的局限性, 不能全方位、正确地反映构筑物, 设计图纸与最终建设成果会有或多或少的误差; 主要存在 的问题如下 ${ }^{[2]}$ : 
（1）设计过程中难免会出现差错

市政路桥设计涉及专业主要有地质勘察、测量、道路、桥梁、隧道、给排水、道路照明等专业, 由于专业较多, 需 要各专业紧密配合, 及时沟通协调; 实际在设计过程中各个专业独立设计, 设计内容无法同步共享, 因此在各专业设计 过程中会出现碰撞与冲突; 沟通协调过程费时费力, 由于人工信息交互准确性没有保证，设计差错的概率也会因此增大。

(2) 施工成果会有误差

设计成果交付后, 建设方还需要组织设计、施工等单位进行合作, 将设计成果转化为实际的道路。由于二维图纸 的局限性, 无法提供直观的道路效果图, 难以让人获得较为深入的理解, 当施工单位对设计理解偏差时会在现场出现 各种各样的问题，不仅浪费钱财，还会耽误进度。

(3) 工作效率有待提高

计算机辅助设计在完成设计后修改较难, 二维设计完成后所需要的效果图、三维展示等只能作为中间成果, 很难 二次利用; 一旦道路方案修改就需要要把所有成果推翻, 进行二次设计。BIM 技术的出现使设计成果的二次利用得以实 现，使设计师工作效率进一步提高。

（4）设计成果与后期各阶段脱节

目前，设计单位并未参与到道路后期运营维护阶段、养护、维修阶段，BIM 技术的优势在于可以通过数字化来协调 建筑信息数据，使之贯穿于项目首末，实现全寿命周期设计。

\section{2 BIM 技术与传统设计方法相比的优点}

(1) 设计流程不同

传统设计道路是在地形图的基础上，依次对道路平面、纵断面、横断面、交叉口、交通工程及其他专业进行设计, 每一步均以前一步为基础, 若前期工作未考虑周全, 就会出现返工且对后期及其他专业影响较大; 而 BIM 技术则是以 模型为核心，将整个道路构建为一个三维信息模型，该模型不仅包含了道路平纵横等道路信息，还囊括了道路路面、 路基、边坡、桥隧构筑物等信息，一方面信息模型可以直观、全面地反应出设计成果，指导施工，作为验收标准，另 一方面这些信息又可以作为媒介与后期施工、运营、养护过程衔接, 实现全生命周期管理的载体。传统设计与 BIM 设 计流程分别如下图 1 所示。

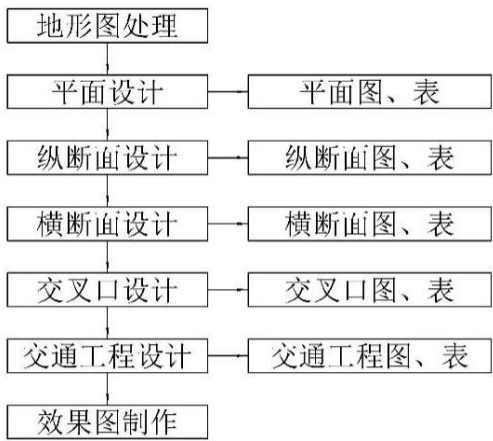

图 1 传统设计过程

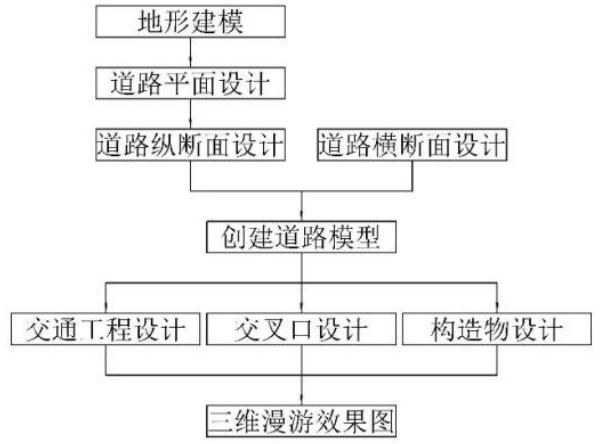

BIM 软件设计过程

（2）信息化、数字化程度不同

设计者一般通过计算机辅助绘图表达自己想法、绘制道桥图纸, 但是设计构思与想法往往受制于二维图纸, 无法 准确表达, 这是由于设计软件数字化与信息化层度较低, 无法将设计成果全面地呈现, 而 BIM 软件则可以通过三维模 型将设计成果表现出来, 而且还可以通过观测模型发现设计过程中的问题, 减少后期返工、变更, 也保证了工程不会 因设计而耽误工期，普通设计图纸（图 2）与三维设计成果（图 3) 如下所示。

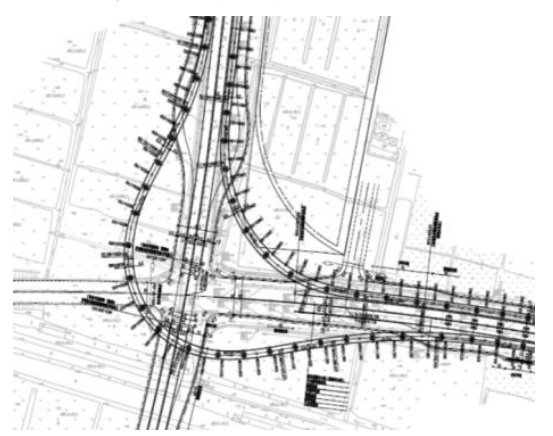

图 2 普通设计图纸
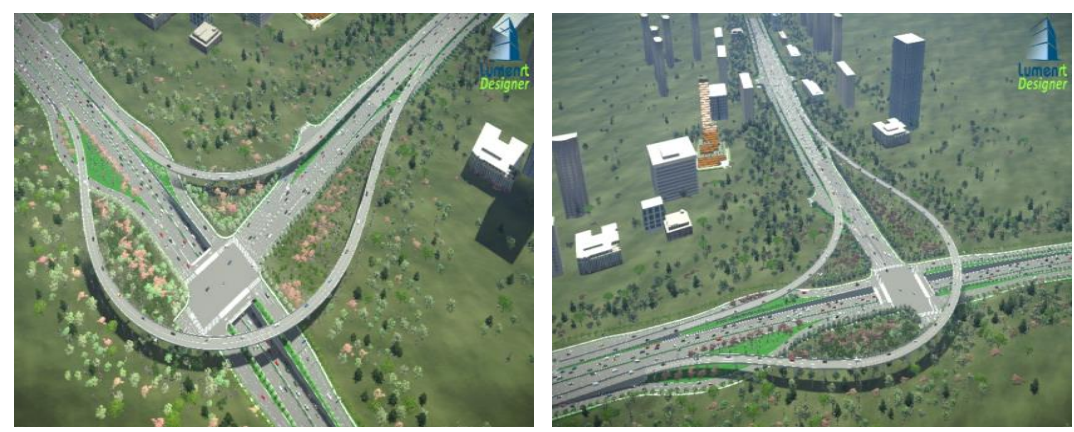

图 3 BIM (Bentley 软件) 设计成果 
使用 Bentley 软件设计出道路模型后, 若需要有变更, 将道路中线修改后道路模型会根据道路中线进行相应调整, 十分方便。

\section{BIM 技术在道路桥梁设计方面的具体应用}

在道路桥梁工程项目的建造设计过程中，有效的应用建筑信息模型技术可以显著的提高道路桥梁工程项目设计方 案的科学性和合理性。及时的发现工程项目设计当中存在的问题和缺陷。保障了道路桥梁工程设计的较高质量和较高 水平。建筑信息模型技术特有的拓展功能也给工程项目的设计工作带来了很大的帮助, 为道路桥梁工程项目的后期建 造施工打下了良好的基础 ${ }^{[5]}$ 。

\section{1 在工程设计数据支持方面应用}

在道路桥梁工程项目的设计工作当中，会使用到大量的工程数据，而这些工程项目的相关数据依赖于前期的工程 勘测工作, 如果工程勘测的工作开展没有严格的根据勘测标准来进行, 将会导致工程勘测的数据结果不可靠、不准确, 这将会造成道路桥梁工程项目的设计方案的缺陷和问题。如果没有能够及时的发现这些问题将会在后期的工程建造施 工中造成严重的质量和安全问题, 甚至产生施工的安全事故。在道路桥梁工程的设计环节应用了建筑信息模型技术就 可以结合工程项目建造标准和设计需要, 将工程项目涉及到的相关数据录入到 BIM 系统当中, 建筑信息模型系统会通 过系统内部设定的建筑信息数据处理功能, 将工程项目的相关数据进行三维立体的展示, 能够更加直观生动地给工程 项目的设计人员展现在当前建造数据的情况下, 工程项目建造施工实际情况, 帮助他们及时的发现工程项目设计方案 当中存在的问题, 并且能够及时的对这些问题进行解决和处理。

Bentley 可以根据需要进行道路模型修改，例如道路绕墩等，具体如下图 4 所示:

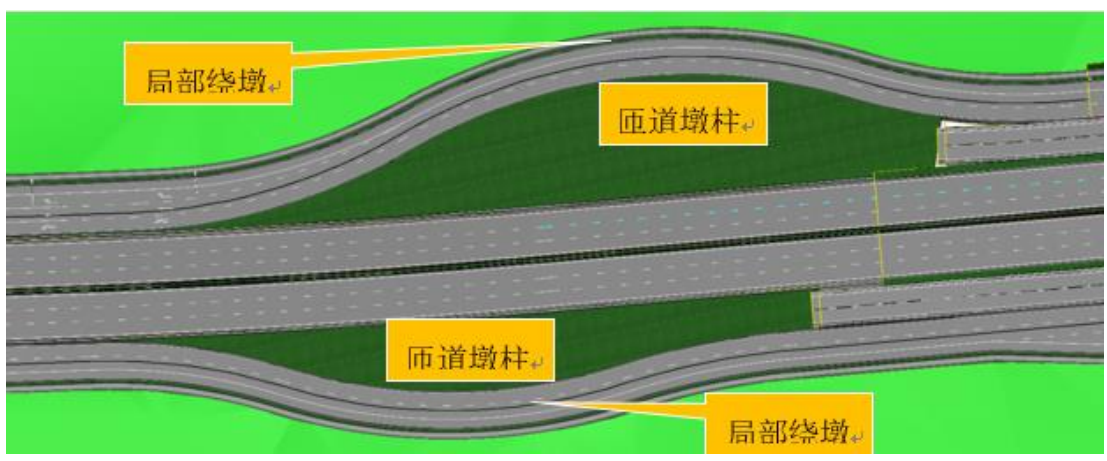

图 4 道路局部绕墩

\section{2 BIM 技术在施工现场分析方面的应用}

道路桥梁工程项目的建造施工会受到施工区域周边的地质地貌, 自然环境, 气候类型等多种因素的影响。在工程 项目的设计过程当中这些因素很难全面、系统、充分的考虑进来, 这将会给道路桥梁工程的项目建造施工以及后期使 用造成很大的影响，而有效的应用建筑信息模型技术在道路桥梁工程的设计当中，可以通过其系统内部的工程虚拟功 能, 更好的对道路桥梁工程项目建造施工区域以及周边的地质地貌, 自然环境, 气候类型等多种影响因素进行科学的 分析。从而更好的完善和修改道路桥梁工程项目的设计方案, 使工程设计方案更加科学、合理, 更符合工程项目建造 的实际情况, 为工程项目的建造质量以及施工安全打下了良好的基础。如果在工程项目的施工环节发现一些设计上的 问题, 再进行工程的返工, 将会提升工程项目的建造施工成本, 降低了工程项目的整体经济效益, 这对于建筑施工企 业来说是非常严重的问题 ${ }^{[6]}$ 。

\section{3 BIM 技术在道路桥梁设计科技研发方面的应用}

在道路桥梁工程设计方面, BIM 技术的应用不仅体现在具体设计环节上, 与科技研发也存在紧密的联系, 将 BIM 技术应用到道路桥梁设计科技研发工作中，能促进科技研发工作的持续稳定开展。因此，在对道路桥梁中心线设计、 三维建模设计、地形图设计及横断面设计进行分析的过程中, 可以加强 BIM 技术的应用, 争取能对各项设计要点进行 优化, 促进设计质量的提高。在具体应用 BIM 技术的过程中, 还要注意对相关设计人员实施积极有效的教育和培训, 为设计人员提供专业的技术指导，确保其可以更好地加强对 BIM 技术的应用，增强科技研发工作的效果。

\section{BIM 技术在路桥设计优化中的一些建议}

\section{1 优化路桥施工模拟, 加快完善数据统计过程}

针对施工技术改进和施工模拟优化而言, 可以在有效利用 BIM 技术的基础上, 对路桥工程的设计方案和施工方案 
进行仔细地检查, 之后对不同区域内的路桥实际情况进行科学地改进、完善与创新。如可以在本着 “开拓创新、与时 俱进” 原则的基础上, 有效地利用 BIM 技术实现施工模拟优化处理的目标, 力求可以最大程度地对路桥工程施工方案 进行科学地完善处理。针对路桥工程施工而言, 施工中的各种数据同样具有非常重要的作用, 所以加强施工数据的运 行管理同样非常关键。 ${ }^{[3]}$ 在道路桥梁工程项目的设计过程当中, 会涉及到大量的工程数据。这些数据的精准、可靠是确 保后续道路桥梁工程项目建造质量和施工安全的重要基础和前提。在道路桥梁工程设计的阶段, 相关设计人员有效的 应用建筑信息模型技术可以更好的保障工程项目数据的准确和可靠, 也能够及时的发现设计方案当中存在的一系列数 据问题，避免在工程项目的施工中由于设计问题而导致工期的延长和施工成本的提升。

\section{2 加强施工技术指导, 积极开展科技研发工作}

在路桥工程建设中应用 BIM 技术, 主要是指导与培训路桥工程施工队伍, 有效地结合以往路桥工程建设中的施工 技术、施工方法等内容, 将路桥工程设计中 BIM 技术的应用专门构成专业的路桥建设实践基地, 由其负责对全体路桥 工程施工人员开展专项教育培训。如除了使施工人员可以深入理解与掌握 BIM 技术及应用方面的理论知识外, 还要注 意培养他们实际应用 BIM 技术的能力, 力求全面提升施工人员的专业技能水平。 ${ }^{\left[{ }^{[5]}\right.}$ 比如, 科技研发工作人员可以有效地 组织全体具有丰富 BIM 技术应用经验的专家现场展示 BIM 技术的应用场景以及过程, 深化施工人员对 BIM 技术及其在 路桥设计中应用方面理论知识的理解, 这种教学方式显著优于传统的科技研发人员沉溺于埋头开发工作中, 而忽视指 导施工人员的效果要好得多, 能够显著提升路桥工程设计人员对 BIM 技术在路桥设计环节和施工环节方面中应用知识 的理解力与运用能力, 有效提升全体设计人员和施工人员运用 BIM 技术的能力。

\section{3 完善行业基本标准, 创新协同化的工作模式}

随着路桥行业的发展, BIM 技术在实际的设计与施工环节中得到了广泛的应用, 同时越来越多的 BIM 技术研发人员 也相应地研发出了许多种不同类型的插件与接口, 这种情况的存在极大地增加了设计人员工作的交流难度。 ${ }^{[6]}$ 为了更好 地提升 BIM 技术在路桥设计和施工中应用的质量, 设计人员要加快从众多 BIM 相关软件技术中选出一些技术水准比较 高的技术, 统一相关插件与接口的输出形式, 尽可能地满足设计人员的设计工作需求。实际的设计中要及时创新 BIM 技术应用中的协同化工作模式，力求不断提升路桥设计质量的同时，可以有效地控制路桥工程建设成本。

\section{5 结束语}

通过上述论证分析, 可以清楚的看到在道路桥梁工程项目的设计环节应用建筑信息模型技术可以产生非常积极和 重要的作用。不仅能够及时的发现和解决设计方案当中存在的一系列问题, 其丰富的拓展功能也为道路桥梁工程设计 方案的整体水平提升打下了良好的基础, 节省了工程项目设计人员工作时间, 提高了设计工作质量, 进一步促进了我 国交通运输行业的整体发展。

\section{[参考文献]}

[1]刘永. BIM 在道路桥梁设计优化中的实践探索 [J]. 建材与装饰, 2019(28): 281-282.

[2]任永明. BIM 关键技术在道路桥梁施工管理中的应用 [J]. 四川建材, 2019 (06) : 194-195.

[3]姬涛. BIM 技术在道路桥梁设计优化方面的应用 [J]. 河南科技, 2018 (26) : 118-119.

[4]安文文. BIM 技术在道路桥梁设计优化方面的应用 [J]. 科技风, 2018 (20): 109.

[5]梁鹏. BIM 技术在道路桥梁设计优化方面的应用 [J]. 四川水泥, 2017 (07) : 125 .

[6]李红涛.试述 BIM 技术在桥梁工程设计优化方面的应用 [J]. 建材与装饰, 2017 (25): 247-248.

作者简介: 王振 (1990.1.1-), 男, 助理工程师, 中国市政工程华北设计研究总院有限公司设计人员。 